\title{
Rotational spectroscopy of isotopic oxirane, $c-\mathrm{C}_{2} \mathrm{H}_{4} \mathrm{O}$
}

\author{
Holger S.P. Müller ${ }^{\mathrm{a}, *}$, Jean-Claude Guillemin ${ }^{\mathrm{b}}$, Frank Lewen ${ }^{\mathrm{a}}$, Stephan Schlemmer ${ }^{\mathrm{a}}$ \\ ${ }^{a}$ Astrophysik/I. Physikalisches Institut, Universität zu Köln, Zülpicher Str. 77, 50937 Köln, Germany \\ ${ }^{b}$ Univ Rennes, Ecole Nationale Supérieure de Chimie de Rennes, CNRS, ISCR-UMR 6226, 35000 Rennes, France
}

\begin{abstract}
We studied the rotational spectrum of oxirane in a sample of natural isotopic composition in selected regions between $158 \mathrm{GHz}$ and $1093 \mathrm{GHz}$. Investigations of the isotopologs with one ${ }^{13} \mathrm{C}$ or one ${ }^{18} \mathrm{O}$ were the primary focus in order to facilitate searches for them in space. We also examined the main isotopic species mainly to look into the performance of Watson's A and S reductions both in an oblate and in a prolate representation. Even though oxirane is a rather asymmetric oblate rotor, the A reduction in the $\mathrm{III}^{l}$ representation did not yield a satisfactory fit, as was observed frequently earlier for other molecules. The other three combinations yielded satisfactory fits of similar quality among each other; the A reduction in the $\mathrm{I}^{r}$ representation required two parameters less than both $\mathrm{S}$ reduction fits.
\end{abstract}

Keywords:

rotational spectroscopy, submillimeter spectroscopy, interstellar molecule, cyclic molecule, centrifugal distortion, reduced Hamiltonian

\section{Introduction}

Oxirane, $c-\mathrm{C}_{2} \mathrm{H}_{4} \mathrm{O}$, also know as ethylene oxide, oxacyclopropane, epoxyethane, and dimethylene oxide, is a molecule of astrochemical interest that was detected first toward the prolific high-mass Galactic center source Sagittarius (Sgr) B2(N) [1]. It was found subsequently in several other high-mass star-forming regions [2, 3]. It was also observed in three rotationally cold, but kinetically warm, Galactic center sources [4] and more recently toward the prototypical low-mass protostellar source IRAS 16293-2422 [5] and toward a prestellar core [6].

Observations of molecules containing ${ }^{13} \mathrm{C}$ are viewed as important diagnostic tools because the ${ }^{12} \mathrm{C} /{ }^{13} \mathrm{C}$ ratio in the interstellar medium (ISM) differs from the terrestrial value of 89 [7]. It is around 20 to 25 in the Galactic center region [8, 9, 10, 11, 12], increases to about 68 in the Solar neighborhood and even further in the outskirts of the Milky Way [8, 12, 13, 14]. Even lower ${ }^{12} \mathrm{C} /{ }^{13} \mathrm{C}$ ratios were found in the envelopes of some late-type stars, such as K4-47 [15, 16].

Numerous detections of isotopologs containing ${ }^{13} \mathrm{C}$ were reported in recent years $[8,11,17,18,12,19]$ and

\footnotetext{
${ }^{*}$ Corresponding author.

Email address: hspm@ph1 . uni-koeln. de (Holger S.P. Müller)
}

some even much earlier. Variations in the ${ }^{12} \mathrm{C} /{ }^{13} \mathrm{C}$ ratios within one source, such as in IRAS 16293-2422 [18], may provide clues on the formation pathways of these molecules. Some of these observations benefited greatly from recent or concomitant laboratory investigations of ${ }^{13} \mathrm{C}$ containing isotopologs, such as ethanol [20], acetaldehyde [21], or ethyl cyanide with two ${ }^{13} \mathrm{C}$ [17]. Other recently studied isotopologs await detection in space. These include methylamine [22], methyl isocyanate [23], methyl mercaptan [24], and cyclopropenone [25].

The relative abundance of ${ }^{18} \mathrm{O}$ is less favorable; the terrestrial ${ }^{16} \mathrm{O} /{ }^{18} \mathrm{O}$ ratio is almost exactly 500 [7], and the ratio in the Galactic center of $\sim 200[11,13,26,27]$ is only slightly lower. Nevertheless, some complex organic molecules containing ${ }^{18} \mathrm{O}$ have been detected in recent years, among them methyl formate [28] and formamide [29]. Cyclopropenone is an example of a molecule detected in the ISM whose ${ }^{18} \mathrm{O}$ isotopolog was studied recently, but was not yet detected [25].

The first studies of the rotational spectrum of oxirane date back to the early days of microwave spectroscopy and include the determination of structural parameters and of its dipole moment through Stark effect measurements [30]. Its dipole moment was determined even earlier through investigations of its dielectric properties along with those of several other molecules [31]. 
More accurate transition frequencies of several isotopic species were determined later in the microwave and lower millimeter-wave regions [32, 33, 34]. Extensive data pertaining to the main isotopolog were obtained more recently between 260 and $360 \mathrm{GHz}$ [35] and between 15 and $73 \mathrm{~cm}^{-1}$ [36]. Results of a millimeterwave and far-infrared study on $c-\mathrm{C}_{2} \mathrm{H}_{3} \mathrm{DO}$ were reported very recently [37]. Unlabeled atoms in formulae designate ${ }^{12} \mathrm{C}$ and ${ }^{16} \mathrm{O}$ here and in the following. We also mention a high-resolution IR study [38] and intensity measurements on $c-\mathrm{C}_{2} \mathrm{H}_{4} \mathrm{O}[39]$.

Our present work describes the investigations of $c{ }^{13} \mathrm{CCH}_{4} \mathrm{O}$ and $c-\mathrm{C}_{2} \mathrm{H}_{4}{ }^{18} \mathrm{O}$ employing samples of oxirane in natural isotopic composition to facilitate their detection in space. We also measured transition frequencies of the main isotopic species mainly to test the performances of Watson's S and A reductions of the rotational Hamiltonian in combination with an oblate and a prolate representation.

\section{Experimental details}

Our measurements were carried out at room temperature using two different spectrometers. Pyrex glass cells with an inner diameter of $100 \mathrm{~mm}$ were employed. Both spectrometer systems used VDI frequency multipliers driven by Rohde \& Schwarz SMF 100A microwave synthesizers as sources. Schottky diode detectors were utilized below $250 \mathrm{GHz}$, whereas a closed cycle liquid Hecooled InSb bolometer (QMC Instruments Ltd) was applied above $340 \mathrm{GHz}$. Frequency modulation was used throughout. The demodulation at $2 f$ causes an isolated line to appear close to a second derivative of a Gaussian, as can be seen in Fig. 1

A $5 \mathrm{~m}$ long double pass cell equipped with Teflon lenses was used for measurements between 158 and $250 \mathrm{GHz}$. The pressures were about $1 \mathrm{~Pa}$. Further information on this spectrometer is available elsewhere [40]. We achieved frequency accuracies of $5 \mathrm{kHz}$ for this spectrometer in a study of 2-cyanobutane [41], a molecule that displays a much richer rotational spectrum. Measurements between 340 and $505 \mathrm{GHz}$ and between 758 and $1093 \mathrm{GHz}$ were carried out employing a $5 \mathrm{~m}$ long single pass cell at pressures of $\sim 2 \mathrm{~Pa}$. A pressure slightly below $1 \mathrm{~Pa}$ was chosen for measurements of medium strong lines of the main isotopolog. Our studies on isotopic formaldehyde [42] or thioformaldehyde [43] demonstrate that accuracies of $10 \mathrm{kHz}$ can be reached quite easily for very symmetric lines with good signal-to-noise ratio up to $1.5 \mathrm{THz}$. We assigned uncertainties of mostly 5 or $10 \mathrm{kHz}$ for the best lines up to
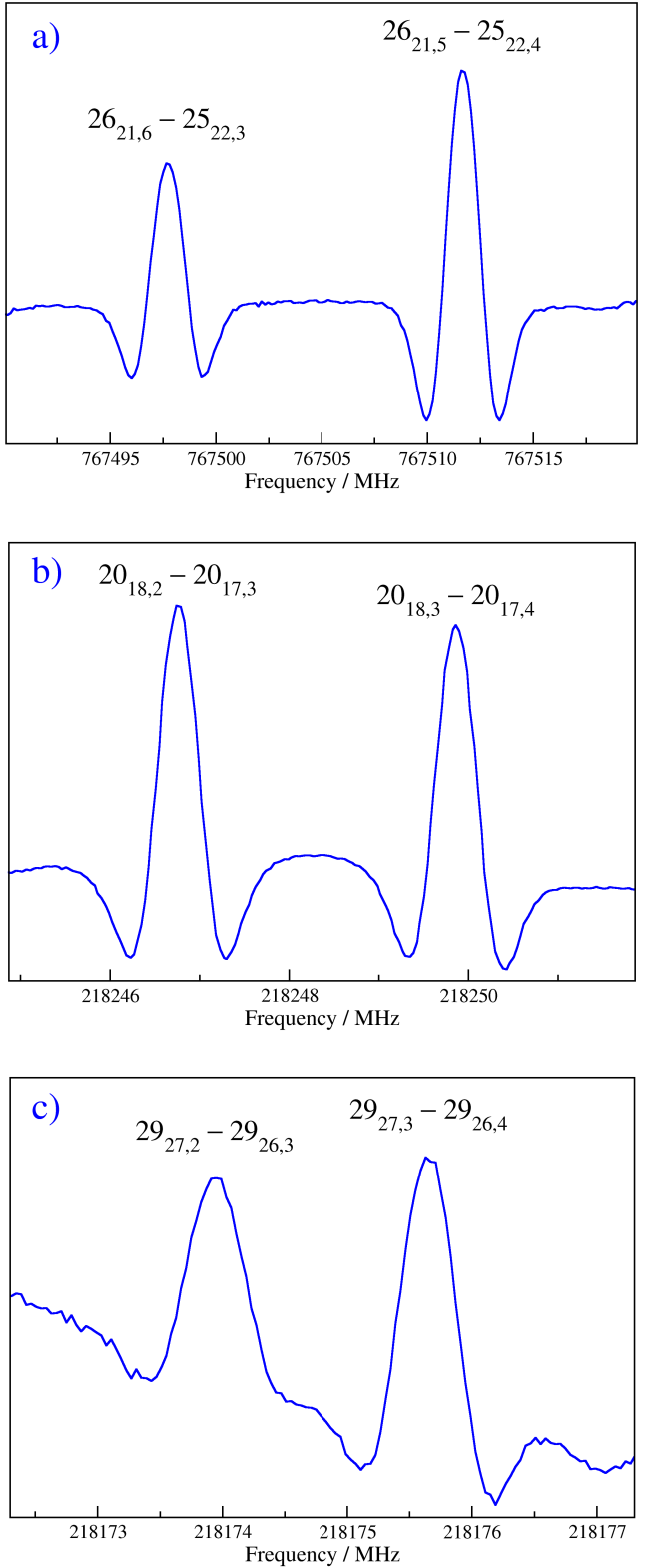

Figure 1: Sections of the rotational spectrum of oxirane displaying the presence of $3: 5$ para to ortho spin-statistics for $c-\mathrm{C}_{2} \mathrm{H}_{4} \mathrm{O}(\mathrm{a})$ and $c-\mathrm{C}_{2} \mathrm{H}_{4}{ }^{18} \mathrm{O}$ (c) as well as the absence in $c_{-}{ }^{13} \mathrm{CCH}_{4} \mathrm{O}$ (b). 
$50 \mathrm{kHz}$ for less symmetric lines or lines fairly close to other lines.

\section{Results}

\subsection{Spectroscopic properties of oxirane}

Oxirane is a very asymmetric rotor of the oblate type with $\kappa=(2 B-A-C) /(A-C)$ equals 0.4093, 0.3557, and 0.6389 for $c-\mathrm{C}_{2} \mathrm{H}_{4} \mathrm{O}, c-{ }^{13} \mathrm{CCH}_{4} \mathrm{O}$, and $c-\mathrm{C}_{2} \mathrm{H}_{4}{ }^{18} \mathrm{O}$, respectively. The main isotopolog as well as the one with ${ }^{18} \mathrm{O}$ have $C_{2 \mathrm{v}}$ symmetry, and the four equivalent $\mathrm{H}$ atoms lead to para and ortho spin-statistics with a $3: 5$ intensity ratio, see Fig. 1. The para and ortho levels are described by $K_{a}+K_{c}$ being odd and even, respectively. These spin-statistics are absent in $c-{ }^{13} \mathrm{CCH}_{4} \mathrm{O}$ because the molecule has only $C_{\mathrm{S}}$ symmetry.

The dipole moment of $1.90 \mathrm{D}$ of $c-\mathrm{C}_{2} \mathrm{H}_{4} \mathrm{O}$ is along the $b$-axis [30]. This value includes a small increase of $0.02 \mathrm{D}$ caused by the difference of the OCS reference value used in [30] compared to more modern values [44, 45]. The lower symmetry of $c_{-}{ }^{13} \mathrm{CCH}_{4} \mathrm{O}$ is associated with a small $a$-dipole moment component of $\sim 0.17 \mathrm{D}$.

The rotational spectrum of oxirane is sparse at the level of the strongest lines because the rotational parameters are fairly large. However, the spectrum is much richer at the level of the weakest transitions in the line list which are about 4.5 orders of magnitude weaker, leading to somewhat increased accidental overlap of lines.

\subsection{Observed spectrum and determination of spectro- scopic parameters of $c-\mathrm{C}_{2} \mathrm{H}_{4} \mathrm{O}$}

The initial calculation of the rotational spectrum of $c-\mathrm{C}_{2} \mathrm{H}_{4} \mathrm{O}$ was based on the second version of the Cologne Database for Molecular spectroscopy, CDMS, [46, 47, 48] entry of the oxirane main isotopic species from July 2012, which, in turn, is based on the FIR study from that year [36]. This study employed Watson's A reduced Hamiltonian in the prolate $\mathrm{I}^{r}$ representation, as was done in the first version of the $c-\mathrm{C}_{2} \mathrm{H}_{4} \mathrm{O}$ CDMS catalog entry of March 2000, on which the FIR study built. The same combination of reduction and representation was used in an IR study [38], whereas Pan et al. employed Watson's A reduction in the oblate $\mathrm{III}^{l}$ representation using 25 spectroscopic parameters in their fit [35]. The initial CDMS fit, in contrast, used only 14 spectroscopic parameters, and the FIR study as well as the second CDMS fit used only one additional spectroscopic parameter.

We scrutinized the existing line lists in order to approach the best possible spectroscopic parameters as closely as possible. The FIR transition frequencies had uncertainties of $0.00020 \mathrm{~cm}^{-1}$ assigned initially [36]. These uncertainties were judged rather conservatively given their rms value was $0.00011 \mathrm{~cm}^{-1}$ in the fit of the FIR study [36]. In addition, one pair of transitions, $J=64-63, K_{c}=62-61$ at $61.468827 \mathrm{~cm}^{-1}$, was omitted from our fit because of a large residual of $\sim 0.0012 \mathrm{~cm}^{-1}$ between measured and calculated frequencies, and one pair of transitions, $J=61-60$, $K_{c}=61-60$ supposedly at $57.435516 \mathrm{~cm}^{-1}$, was reassigned to a much stronger transition $\left(37_{26,12}-36_{25,11}\right)$ much closer to the calculated position. The FIR rms was reduced to $0.00009 \mathrm{~cm}^{-1}$ after these two corrections, and we assigned $0.00010 \mathrm{~cm}^{-1}$ as uncertainties to the FIR transition frequencies. The assignments of four more transition frequencies were extended eventually because more individual transitions contributed substantially to each measured line than was indicated in the initial line list [36]; further details are available in our line list which is part of the Supplementary Material. These corrections, however, had only very small effects on the fit. It is important in this context to emphasize that in the case of two or more overlapping transitions, our fitting program SPFIT only judges the intensity-weighted average position of all overlapping transitions.

One set of MW transition frequencies [34] was reportedly accurate to $0.01 \mathrm{MHz}$, but several lines displayed larger residuals between calculated and measured frequencies. In fact, the rms of this data set was very close to $0.02 \mathrm{MHz}$, and we attributed this value to this set of transition frequencies. Uncertainties of $0.05 \mathrm{MHz}$ and $0.15 \mathrm{MHz}$, respectively, were employed for the remaining datasets [33, 35], as was done earlier [36].

Spectroscopic parameters were determined for this as well as various intermediate line lists using the A reduction in the $\mathrm{I}^{r}$ representation and employing the $\mathrm{S}$ reduction in the $\mathrm{III}^{l}$ and $\mathrm{I}^{r}$ representations. The A reduction in the $\mathrm{III}^{l}$ representation was only used for the final line list as this combination of reduction and representation appeared to require many more spectroscopic parameters than the other combinations. Pickett's SPFIT program [49] was applied for all fitting, and the SPCAT program was utilized for calculations of all rotational spectra.

We recorded individual transitions or pairs thereof if transitions happened to be close in frequency throughout our study. Series in particular combinations of $K_{a}$ and $K_{c}$ were followed in all instances. Asymmetry doublets with significant, but not complete splitting were avoided as their positions are usually determinable with less accuracy compared to well collapsed or well re- 
Table 1: Spectroscopic parameters ${ }^{a}(\mathrm{MHz})$ of the main isotopolog of oxirane employing Watson's $\mathrm{S}$ and A reduction of the rotational Hamiltonian both in the oblate representation $\mathrm{III}^{l}$ and in the prolate representation $\mathrm{I}^{r}$.

\begin{tabular}{|c|c|c|c|c|c|}
\hline \multicolumn{3}{|c|}{$\mathrm{S}$ reduction } & \multicolumn{3}{|c|}{ A reduction } \\
\hline Parameter & $\mathrm{IIII}^{l}$ & $\mathrm{I}^{r}$ & $\mathrm{I}^{r}$ & $\mathrm{IIII}^{l}$ & Parameter \\
\hline$A$ & $25483.88987(11)$ & $25483.88751(10)$ & $25483.86273(9)$ & $25483.88386(10)$ & $A$ \\
\hline$B$ & 22120.83638 (9) & $22120.82714(9)$ & $22120.87303(7)$ & $22120.84394(9)$ & $B$ \\
\hline$C$ & $14097.83590(11)$ & $14097.85210(11)$ & $14097.82581(8)$ & $14097.83400(16)$ & $C$ \\
\hline$D_{K} \times 10^{3}$ & $26.07490(13)$ & $2.95932(23)$ & $27.58930(22)$ & $27.63421(33)$ & $\Delta_{K} \times 10^{3}$ \\
\hline$D_{J K} \times 10^{3}$ & $-68.65174(30)$ & $50.46597(26)$ & $20.91041(21)$ & $-70.50171(68)$ & $\Delta_{J K} \times 10^{3}$ \\
\hline$D_{J} \times 10^{3}$ & $50.83976(24)$ & $15.75678(24)$ & $20.68240(14)$ & $51.14486(26)$ & $\Delta_{J} \times 10^{6}$ \\
\hline$d_{1} \times 10^{3}$ & 9.02067 (5) & $-6.21001(6)$ & $18.10824(9)$ & $3.39130(43)$ & $\delta_{K} \times 10^{3}$ \\
\hline$d_{2} \times 10^{3}$ & $-0.15233(2)$ & $-2.46284(1)$ & $6.21005(2)$ & $-9.01957(5)$ & $\delta_{J} \times 10^{3}$ \\
\hline$H_{K} \times 10^{9}$ & $-191.41(11)$ & $2712.32(23)$ & $2.2415(2)$ & $-10.8512(10)$ & $\Phi_{K} \times 10^{6}$ \\
\hline$H_{K J} \times 10^{9}$ & $373.61(21)$ & $-3535.95(28)$ & $-2.6387(3)$ & $16.0605(16)$ & $\Phi_{K J} \times 10^{6}$ \\
\hline$H_{J K} \times 10^{9}$ & $-198.52(24)$ & $1096.22(23)$ & $624.58(18)$ & $-5321.27(97)$ & $\Phi_{J K} \times 10^{9}$ \\
\hline$H_{J} \times 10^{9}$ & $17.92(19)$ & $-46.58(18)$ & $-1.797(69)$ & $110.91(22)$ & $\Phi_{J} \times 10^{9}$ \\
\hline$h_{1} \times 10^{9}$ & $-8.211(44)$ & $5.268(45)$ & $-0.3278(1)$ & $-18.0197(18)$ & $\phi_{K} \times 10^{6}$ \\
\hline$h_{2} \times 10^{9}$ & $46.364(24)$ & $22.470(14)$ & $277.30(10)$ & $1546.63(67)$ & $\phi_{J K} \times 10^{9}$ \\
\hline$h_{3} \times 10^{9}$ & $-49.529(4)$ & $-6.868(3)$ & $-1.730(7)$ & $-57.773(50)$ & $\phi_{J} \times 10^{9}$ \\
\hline$L_{K} \times 10^{12}$ & $-3.325(25)$ & $-14.450(79)$ & $-0.62(9)$ & $-132.5(38)$ & $L_{K} \times 10^{12}$ \\
\hline$L_{K K J} \times 10^{12}$ & $8.202(86)$ & $26.454(117)$ & $-6.79(14)$ & $177.5(61)$ & $L_{K K J} \times 10^{12}$ \\
\hline$L_{I K} \times 10^{12}$ & $-8.000(98)$ & $-13.601(102)$ & $8.18(11)$ & $-33.5(23)$ & $L_{I K} \times 10^{12}$ \\
\hline$L_{J J K} \times 10^{12}$ & $4.200(57)$ & $-1.218(58)$ & $-3.00(5)$ & $-9.36(40)$ & $L_{J J K} \times 10^{12}$ \\
\hline$L_{J} \times 10^{12}$ & $-1.083(49)$ & 0.469 (44) & & $-1.31(6)$ & $L_{J} \times 10^{12}$ \\
\hline$l_{1} \times 10^{15}$ & $479.6(119)$ & $117.3(99)$ & $-13.00(4)$ & $-207.8(48)$ & $l_{K} \times 10^{12}$ \\
\hline$l_{2} \times 10^{15}$ & $-72.7(81)$ & $-297.1(36)$ & $8.07(5)$ & $-110.5(14)$ & $l_{K J} \times 10^{12}$ \\
\hline$l_{3} \times 10^{15}$ & $87.5(25)$ & $-147.8(15)$ & $-2.42(3)$ & $4.43(26)$ & $l_{J K} \times 10^{12}$ \\
\hline$l_{4} \times 10^{15}$ & $-11.9(4)$ & $31.7(2)$ & & $0.561(14)$ & $l_{J} \times 10^{12}$ \\
\hline & & & & $-187.5(9)$ & $P_{K} \times 10^{15}$ \\
\hline & & & & $361.2(30)$ & $P_{K K J} \times 10^{15}$ \\
\hline & & & & $-204.5(33)$ & $P_{K J} \times 10^{15}$ \\
\hline & & & & $30.7(10)$ & $P_{J K} \times 10^{15}$ \\
\hline & & & & $-33.9(9)$ & $p_{K} \times 10^{15}$ \\
\hline & & & & $90.7(23)$ & $p_{K K J} \times 10^{15}$ \\
\hline & & & & $-12.7(3)$ & $p_{J K} \times 10^{15}$ \\
\hline \# trans. ${ }^{b, c}$ & 2942 & 2942 & 2942 & 2942 & $\#$ trans. ${ }^{b, c}$ \\
\hline$\#$ new tr. ${ }^{b, c}$ & 1091 & 1091 & 1091 & 1091 & \# new $\operatorname{tr}^{b, c}$ \\
\hline$J_{\max } b, c$ & 63 & 63 & 63 & 63 & $J_{\max } b, c$ \\
\hline$K_{a, \max }^{b, c}$ & 43 & 43 & 43 & 43 & $K_{a, \max }^{b, c}$ \\
\hline$K_{c, \max }^{b, c}$ & 61 & 61 & 61 & 61 & $K_{c, \max }^{b, c}$ \\
\hline $\mathrm{wrms}^{b, c}$ & 0.940 & 0.957 & 0.946 & 1.162 & $\mathrm{wrms}^{b, d}$ \\
\hline
\end{tabular}

${ }^{a}$ Numbers in parentheses are one standard deviation in units of the least significant figures.

${ }^{b}$ Dimensionless.

${ }^{c}$ See Section 3.2 for additional details on the numbers of transitions and the maximum quantum numbers.

${ }^{d}$ Weighted standard deviation of the fit. 
solved asymmetry doublets. The strongest transitions of the main isotopolog were avoided because their uncertainties were already small such that their impact in the fit would be small. Moreover, these transitions are affected by opacity issues.

The 340-506 GHz region was studied extensively with 842 transitions in the final line list. Additional measurements were made between $160-250 \mathrm{GHz}$ and 764-1046 GHz, where 61 and 188 further transitions, respectively, were recorded and retained in the final line list. The number of different frequencies is $727 ; 728$ transitions represent unresolved asymmetry doublets. The maximum quantum numbers $J, K_{a}$, and $K_{c}$ of our new transition frequencies are 55,43 , and 52, respectively.

The newly recorded transition frequencies deviated by modest amounts from the calculations based on the previous FIR study [36], the largest deviation was 4.32 MHz. The deviations were largest for transitions with high values of $J$ and with $K_{c}=J$. The rms of the new lines fit with the old parameters was $580 \mathrm{kHz}$, which corresponded to 38 times the experimental uncertainties on average. The signed deviation was $15 \mathrm{kHz}$, which means that deviations to higher frequencies were only marginally more important than deviations to lower frequencies.

We test the need for additional spectroscopic parameters usually by adding one parameter at a time and evaluating which of the new parameters improves the fit the most. This procedure ensures that the parameter set is about as small as possible and that it is fairly unique. The procedure works usually very well for prolate rotors, though correlation may in some cases require sets of two parameters to be tested. Fitting oblate type rotors was often less straightforward. In all combinations of reductions and representations we tested sets of two parameters to fit the $c-\mathrm{C}_{2} \mathrm{H}_{4} \mathrm{O}$ transition frequencies; we even tested sets of three and four parameters in the case of the A reduction in the $\mathrm{III}^{l}$ representation.

The previous FIR study [36] employed 15 parameters using the A reduction in the $\mathrm{I}^{r}$ representation; these were a nearly complete set of parameters up to sixth order, except $\Phi_{J}$, plus $l_{K}$. Our corresponding new fit consists of seven more parameters and is described as a nearly complete set of parameters up to eighth order, except $L_{J}$ and $l_{J}$, as summarized in Table 1 . The weighted rms (or rms error) of the fit is 0.946 with modest scatter for the individual data sets: 1.087 and 1.057 for 19 [34] and 40 microwave lines [33], respectively; 1.000 for 607 millimeter and submillimeter transitions [35]; 0.872 for 1185 FIR transitions [36]; and 0.957 for our 1091 transitions with an rms of $20.3 \mathrm{kHz}$.
The quality of the fits utilizing the $\mathrm{S}$ reduction in the III $^{l}$ or the $\mathrm{I}^{r}$ representation were similar, but required two parameters more in most fits; the difference was in some intermediate fits larger, in few smaller. The final spectroscopic parameters are also listed in Table 1 . We tested the predictive power of the three parameter sets occasionally and found that there was no persistent preference for either set.

Obtaining a somewhat satisfactory fit applying the A reduction in the III $^{l}$ representation with the final line list was challenging. A complete set of parameters up to the eighth order plus several decic parameters yielded a fit that had an rms error more than $20 \%$ worse than any of the other fits; the parameters of this fit are also given in Table 1. We tested all reasonable parameters individually, all or nearly all combinations of two of these along with several combinations of three and even some four parameter combinations. These improved the rms error only marginally, $\sim 1.12$ was the best we were able to achieve. This modest refinement was discarded as it came at the expense of many more spectroscopic parameters.

\subsection{Observed spectrum and determination of spectro- scopic parameters of $\mathrm{c}_{-}{ }^{13} \mathrm{CCH}_{4} \mathrm{O}$ and $\mathrm{c}_{-} \mathrm{C}_{2} \mathrm{H}_{4}{ }^{18} \mathrm{O}$}

Only 11 and 15 transitions were reported by Creswell and Schwendeman for $c{ }^{13} \mathrm{CCH}_{4} \mathrm{O}$ and $c-\mathrm{C}_{2} \mathrm{H}_{4}{ }^{18} \mathrm{O}$, respectively. The uncertainties were reportedly $10 \mathrm{kHz}$, a value that was used in early fits, but later increased to $20 \mathrm{kHz}$ because of the rms of these data, as in the case of the main isotopolog, see section 3.2. Data reported by Hirose [32] were not considered initially even though slightly more transition frequencies were reported extending to slightly higher quantum numbers. The reasons were the larger uncertainties and in part large residuals between measured and calculated frequencies already in the publication, especially for $c-\mathrm{C}_{2} \mathrm{H}_{4}{ }^{18} \mathrm{O}$.

The number of transition frequencies was small for both isotopologs. Therefore, the spectroscopic parameters of the main isotopic species in the A reduction and $\mathrm{I}^{r}$ representation from the previous FIR study [36] were taken as starting values for both isotopic species. The rotational parameters were adjusted in a first step. Through several trial fits, $\delta_{K}$ and $\delta_{J}$ were determined as the parameters which improved the rms error of the $c-{ }^{13} \mathrm{CCH}_{4} \mathrm{O}$ fit the most. The corresponding parameters for $c-\mathrm{C}_{2} \mathrm{H}_{4}{ }^{18} \mathrm{O}$ were also $\delta_{K}$ and $\delta_{J}$ and additionally $\Delta_{J K}$. Minor adjustments of some further parameters, as frequently done [25, 42, 43], improved the fit only slightly.

We started our investigations of the two minor isotopic species in the $340-505 \mathrm{GHz}$ region followed by 
$158-250 \mathrm{GHz}$ and $758-1092 \mathrm{GHz}$, as for the main isotopic species. There was no caution required to avoid strong transitions of these isotopologs as their abundances in natural isotopic composition are lower than those of the main species by factors of $\sim 45$ (there are two structural identical $\mathrm{C}$ atoms in the molecule) and $\sim 500$, respectively. The quality of the extrapolations to submillimeter wavelengths was unclear because of the limited data set. Moreover, only one $R$-branch transition frequency was reported for either isotopolog, and this was the $J=1-0$ transition. Since patterns of two transitions are easier to recognize than individual transitions, we searched for transitions with small, but resolved asymmetry splitting. In the case of $\mathrm{c}^{13} \mathrm{CCH}_{4} \mathrm{O}$, these were transitions with $K_{a}=J$ and $J^{\prime \prime}=9,8$, and 7 as well as transitions having $K_{c}=J-4$ and $J^{\prime \prime}=14,13$, and 12 . Both types of transitions were also searched for in the case of $c-\mathrm{C}_{2} \mathrm{H}_{4}{ }^{18} \mathrm{O}$, but with slightly different values of $J^{\prime \prime}$ because of the very different $\kappa$ value, see section 3.1 , namely 10 to 7 for the nearly prolate paired transitions and 11 to 9 for the nearly oblate paired transitions plus the oblated paired $J^{\prime \prime}=14$ in this series. After almost all of these transitions were found to be unblended, the spectroscopic parameters of both isotopologs were updated, and more transitions with similar combinations of quantum numbers were recorded. Subsequently, further series were sought, including large series of $Q$-branch transitions until there were no more transitions that had enough intensity to be recorded and a calculated uncertainty at least of order of the achievable measurement accuracy.

The final line lists consisted of 553 and 274 transitions between $340-505 \mathrm{GHz}, 211$ and 186 transitions between 158-250 GHz, and 341 and 207 transitions between 758-1092 GHz for the isotopologs containing one ${ }^{13} \mathrm{C}$ and one ${ }^{18} \mathrm{O}$, respectively. 680 of the 1111 and 468 of the 667 transitions correspond to unresolved asymmetry doublets for $c-{ }^{13} \mathrm{CCH}_{4} \mathrm{O}$ and $c-\mathrm{C}_{2} \mathrm{H}_{4}{ }^{18} \mathrm{O}$, respectively, resulting in 771 and 433 different transition frequencies. The maximum quantum numbers $J, K_{a}$, and $K_{c}$ of our new transition frequencies are 45, 34, and 39 for $c{ }^{13}{ }^{13} \mathrm{CH}_{4} \mathrm{O}$ and 38,30 , and 38 for $c-\mathrm{C}_{2} \mathrm{H}_{4}{ }^{18} \mathrm{O}$.

As the A reduction in the $\mathrm{I}^{r}$ representation required the least number of spectroscopic parameters to fit the transition frequencies of the main isotopic species, we only tried this combination of reduction and representation. Additional parameters for the main isotopolog were subsequently transferred as fixed parameters to the fits of the minor isotopologs. Higher order distortion parameters of these, which were kept fixed in the fit, were adjusted if lower order parameters differed markedly from those of the main isotopic species. The changes
Table 2: Spectroscopic parameters ${ }^{a}(\mathrm{MHz})$ of the oxirane isotopologs containing one ${ }^{13} \mathrm{C}$ or one ${ }^{18} \mathrm{O}$.

\begin{tabular}{|c|c|c|}
\hline Parameter & $c-{ }^{13} \mathrm{CCH}_{4} \mathrm{O}$ & $c-\mathrm{C}_{2} \mathrm{H}_{4}{ }^{18} \mathrm{O}$ \\
\hline$A$ & $25291.99778(17)$ & $23992.43450(20)$ \\
\hline$B$ & 21597.97459 (13) & 22121.17197 (14) \\
\hline$C$ & 13825.77925 (14) & $13628.24006(9)$ \\
\hline$\Delta_{K} \times 10^{3}$ & $27.62104(47)$ & $24.65010(70)$ \\
\hline$\Delta_{J K} \times 10^{3}$ & $20.47198(31)$ & 17.45625 (69) \\
\hline$\Delta_{J} \times 10^{6}$ & $20.03716(31)$ & $20.48033(8)$ \\
\hline$\delta_{K} \times 10^{3}$ & $18.29406(30)$ & $16.34518(30)$ \\
\hline$\delta_{J} \times 10^{3}$ & $6.05179(5)$ & $6.31363(4)$ \\
\hline$\Phi_{K} \times 10^{6}$ & $1.9510(4)$ & $2.0748(8)$ \\
\hline$\Phi_{K J} \times 10^{6}$ & $-2.2614(6)$ & $-2.4735(9)$ \\
\hline$\Phi_{J K} \times 10^{6}$ & $0.5141(2)$ & $0.5999(6)$ \\
\hline$\Phi_{J} \times 10^{9}$ & $4.97(30)$ & -1.9 \\
\hline$\phi_{K} \times 10^{6}$ & $-0.2208(6)$ & $-0.3603(3)$ \\
\hline$\phi_{J K} \times 10^{6}$ & $0.2292(5)$ & $0.2704(3)$ \\
\hline$\phi_{J} \times 10^{9}$ & $-0.251(29)$ & -1.6 \\
\hline$L_{K} \times 10^{12}$ & -0.51 & -0.56 \\
\hline$L_{K K J} \times 10^{12}$ & $-7.42(37)$ & -6.56 \\
\hline$L_{J K} \times 10^{12}$ & 7.00 & 7.40 \\
\hline$L_{J J K} \times 10^{12}$ & -2.40 & -2.48 \\
\hline$L_{J} \times 10^{12}$ & $-1.158(97)$ & \\
\hline$l_{K} \times 10^{12}$ & $-12.66(35)$ & -10.1 \\
\hline$l_{K J} \times 10^{12}$ & $9.45(45)$ & 7.0 \\
\hline$l_{J K} \times 10^{12}$ & $-3.12(21)$ & -2.5 \\
\hline \multicolumn{3}{|l|}{$l_{J} \times 10^{12}$} \\
\hline \# trans. ${ }^{b, c}$ & 1137 & 701 \\
\hline \# new tr. ${ }^{b, c}$ & 1111 & 667 \\
\hline$J_{\max }^{b, c}$ & 45 & 38 \\
\hline$K_{a, \max }^{b, c}$ & 34 & 30 \\
\hline$K_{c, \max }^{b, c}$ & 39 & 38 \\
\hline $\mathrm{wrms}^{b, d}$ & 0.907 & 0.906 \\
\hline
\end{tabular}

${ }^{a}$ Watson's A reduction was used in the $\mathrm{I}^{r}$ representation. Numbers in parentheses are one standard deviation in units of the least significant figures. Spectroscopic parameters without uncertainties were evaluated from the main isotopic species and kept fixed in the fits, see section 3.3

${ }^{b}$ Dimensionless.

${ }^{c}$ See Section 3.3 for additional details on the numbers of transitions and the maximum quantum numbers.

${ }^{d}$ Weighted standard deviation of the fit. 
were applied by evaluating trends in related parameters, such as $\Delta_{K}, \Phi_{K}$, and $L_{K}$.

Our new transition frequencies of the two minor isotopic species deviated much more from the initial calculations than in the case of the main isotopolog because of the much smaller initial line lists for both minor isotopologs which resulted in substantially less reliable spectroscopic parameters. The rms of the new lines fit with the initial parameters is $29.9 \mathrm{MHz}$, the average shift is $17.3 \mathrm{MHz}$, and the rms corresponds to more than 2500 times the experimental uncertainties on average for $c{ }^{13} \mathrm{CCH}_{4} \mathrm{O}$. In the case of $c-\mathrm{C}_{2} \mathrm{H}_{4}{ }^{18} \mathrm{O}$, that rms is 63.4 MHz, the average shift is $-14.5 \mathrm{MHz}$, and the rms corresponds to almost 3450 times the experimental uncertainties on average.

Spectroscopic parameters derived from the main isotopolog or new spectroscopic parameters were tested as was done for the main isotopic species. That is, we searched for the parameter whose floating or inclusion improved the fit the most in each fitting round. An additional prerequisite to keep a new parameter in the fit or to keep a previously fixed parameter floated was that the parameter was determined sufficiently well, meaning that its uncertainty should be less than one fifth of its magnitude.

In the final fitting round, we tested the transition frequencies reported by Hirose [32]; 15 and 18 lines were included with uncertainties of $0.05 \mathrm{MHz}$ for $c-{ }^{13} \mathrm{CCH}_{4} \mathrm{O}$ and $c-\mathrm{C}_{2} \mathrm{H}_{4}{ }^{18} \mathrm{O}$, respectively, and three respectively four lines were omitted because of large residuals. The final sets of spectroscopic parameters are given in Table 2

The weighted rms (or rms errors) of the fits are 0.907 and 0.906 for the isotopologs containing one ${ }^{13} \mathrm{C}$ and one ${ }^{18} \mathrm{O}$, respectively. The rms errors for 11 lines from Creswell and Schwendemann [34], 15 lines from Hirose [32], and 1111 lines from our present study are $0.898,0.805$, and 0.909, respectively for $c^{-13} \mathrm{CCH}_{4} \mathrm{O}$; the rms of our lines is $22.1 \mathrm{kHz}$. The corresponding values for 16 lines from Creswell and Schwendemann [34], 18 lines from Hirose [32], and 667 lines from our present study are $1.056,0.978$, and 0.897 , respectively; the rms of our lines is $24.4 \mathrm{kHz}$.

\section{Discussion}

The main interest for our study of the oxirane main isotopolog was the test of various combinations of reduction and representation. We point out that we did not have any program at our disposal to try out the $\mathrm{II}^{r}$ or $\mathrm{II}^{l}$ representation, but these are very rarely used in published fits anyhow. A rather large set of spectroscopic parameters was needed to obtain a somewhat satisfactory fit in the $\mathrm{III}^{l}$ representation using the A reduction, similar to results from a millimeter and submillimeter study [35]. This combination of reduction and representation would be considered by many spectroscopists to be the natural choice as they commonly use the A reduction for traditional reasons and as oxirane is an asymmetric top of the oblate type that is with $\kappa=0.4093$ quite far from the symmetric limit.

Considerably fewer parameters, however, are required in the $\mathrm{III}^{l}$ representation using the $\mathrm{S}$ reduction, as can be seen in Table 1, emphasizing again the greater versatility of the $S$ reduction compared to the A reduction if the representation is used that fits to the asymmetry type of the molecule. We may, of course, also use a different representation. The $\mathrm{S}$ reduction in the $\mathrm{I}^{r}$ representation yields a fit of similar quality and with the same number of parameters as the S reduction in the III $^{l}$ representation. It is interesting to note that the A reduction in the $\mathrm{I}^{r}$ representation requires two parameters fewer still to produce a fit of about the same quality. Thus, while the $S$ reduction is the prime choice if one only considers the III $^{l}$ representation, the A reduction in the $\mathrm{I}^{r}$ representation is preferable if all these four combinations are considered.

The poor performance of the A reduction in an oblate representation (III ${ }^{l}$ and III $^{r}$ differ only in some signs of off-diagonal distortion parameters) is not unique to oxirane, but is at least somewhat more widespread. Yamada and Klee [50] carried out an FIR study on $\mathrm{H}_{2} \mathrm{~S}$ and fit their data together with rotational data of microwave accuracy, employing full parameter sets of eighth order and diagonal decic parameters. Although $\mathrm{H}_{2} \mathrm{~S}$ is an asymmetric rotor of the oblate type $(\kappa=0.5234)$, they encountered convergence problems using the A reduction in the III $^{r}$ representation. A satisfactory fit resulted from a fit in which the $S$ reduction in the same representation was applied. The authors also tried fits in the $\mathrm{I}^{r}$ representation. In this case, the A reduction gave a fit that was noticeably worse than the S and III $^{r}$ combination, whereas the $\mathrm{S}$ reduction (in $\mathrm{I}^{r}$ ) yielded the best fit.

Another example is the lowest energy conformer of 2-cyanobutane, which is a very asymmetric rotor of the oblate type $\kappa=0.1404$ [41]. The combination of A reduction and $\mathrm{III}^{l}$ representation required the most parameters; the best result was achieved with the $\mathrm{S}$ reduction in the III $^{l}$ representation while both reductions in the $\mathrm{I}^{r}$ representation required an intermediate number of parameters.

In a study of $\mathrm{SO}_{2}$ isotopologs containing one and two ${ }^{18} \mathrm{O}$, asymmetric top molecules somewhat close to the 
prolate limit, Margulès et al. [51] reemphasized Watson's recommendation to favor the $\mathrm{S}$ reduction over the A reduction because of the smaller correlation coefficients [52]. The authors added that the $\mathrm{S}$ reduction may be a better choice even in cases in which more spectroscopic parameters are needed because of the more favorable condition numbers [51]. The condition number is the ratio of the largest singular value of the Jacobian matrix over the smallest and indicates the degree of ill-conditioning; a large value may indicate that the determined spectroscopic parameters are not reliable. Among the condition numbers of the fits of the main isotopolog, the value is very large, almost 15000 , only in the case of the $\mathrm{III}^{l}$ representation and the A reduction; the values are much smaller and fairly similar, 150, 91, and 92 , for the combinations $\mathrm{III}^{l} \& \mathrm{~S}, \mathrm{I}^{r} \& \mathrm{~A}$, and $\mathrm{I}^{r} \&$ $\mathrm{S}$, respectively. Therefore, our preference of $\mathrm{I}^{r} \& \mathrm{~A}$ is supported from the viewpoint of the condition number.

Substitution of one ${ }^{12} \mathrm{C}$ with ${ }^{13} \mathrm{C}$ or of one ${ }^{16} \mathrm{O}$ with ${ }^{18} \mathrm{O}$ reduces the rotational parameters by modest amount, except for $B$ of the $c-\mathrm{C}_{2} \mathrm{H}_{4}{ }^{18} \mathrm{O}$ isotopolog. Substitution of an atom on the symmetry axis does not affect the equilibrium rotational parameter in the Born-Oppenheimer approximation. The corresponding ground state rotational parameter of a heavy-atom substitution is frequently slightly larger because of effects of anharmonicity, as is observed in the case of oxirane. The quartic distortion parameters of both minor isotopic species are quite close to those of the main species, those of the ${ }^{13} \mathrm{C}$ species more so than those of the ${ }^{18} \mathrm{O}$, which is commensurate with the changes in rotational parameters. The situation is more complex among the higher order parameters, in part possibly because fewer parameters were varied as a consequence of the smaller data sets extended to lower quantum numbers. Some parameters of $c_{-}{ }^{13} \mathrm{CCH}_{4} \mathrm{O}$ display large changes compared to the main isotopolog, possibly as a consequence of the different symmetry. The $\Phi_{J}$ value of the main species appears to be accidentally very small in magnitude compared with almost all of the remaining sextic distortion parameters. The $\Phi_{J}$ value of $c-{ }^{13} \mathrm{CCH}_{4} \mathrm{O}$ is larger in magnitude and has an opposite sign; it was even necessary to fit the related $L_{J}$ for this isotopolog. On the other hand, $\phi_{J}$ is much smaller in magnitude than that of the main species. The values of some of the octic distortion parameters will depend on the values of parameters that were kept fixed in the fit.

We were interested if $c_{-}{ }^{13} \mathrm{CCH}_{4} \mathrm{O}$, as the more promising of the two minor isotopologs, could be found in existing astronomical data. The ${ }^{12} \mathrm{C} /{ }^{13} \mathrm{C}$ ratio is particularly favorable in the Galactic center. Among its sources, Sagittarius (Sgr) B2 is among the most promi- nent sources in the whole Galaxy. A search toward Sgr B2(N1S) and Sgr B2(N2) in the ReMoCA data, a molecular line survey at $3 \mathrm{~mm}$ with the Atacama Large Millimeter Array [29], was unsuccessful. Assuming a ${ }^{12} \mathrm{C} /{ }^{13} \mathrm{C}$ ratio of 20 [9, 10, 11], all lines of $c-{ }^{13} \mathrm{CCH}_{4} \mathrm{O}$ were heavily blended with other, stronger lines or did not exceed the $3 \sigma$ average noise level of the survey (A. Belloche, private communication, 2021).

\section{Conclusion}

We have obtained greatly improved spectroscopic parameters for the $c{ }^{13} \mathrm{CCH}_{4} \mathrm{O}$ and $c-\mathrm{C}_{2} \mathrm{H}_{4}{ }^{18} \mathrm{O}$ isotopologs which should be sufficiently accurate for all radio astronomical observations. The investigations into the performance of various combinations of representations and reductions in fitting an extended data set of the oxirane main isotopolog revealed once more the poor performance of the A reduction in an oblate representation, suggesting that these combinations should be avoided. In the more general case of oblate- as well as prolatetype asymmetric rotors we conclude that the $S$ reduction should be preferred for fits employing a natural choice of representation. Other choices are only recommended if all common combinations of representation and reduction were tested.

Concerning $c-{ }^{13} \mathrm{CCH}_{4} \mathrm{O}$ in space, it appears as if dedicated searches are required to detect this isotopolog in the interstellar medium.

\section{Note added in proof}

After acceptance of our manuscript, we became aware of an interesting article on dimethylsulfoxide [53] that also discusses reductions and representations. The findings are in agreement with the findings in publications discussed in our article.

\section{CRediT authorship contribution statement}

Holger S.P. Müller: Conceptualization, Investigation, Methodology, Formal analysis, Validation, Data curation, Writing - Original Draft, Writing - review \& editing. Jean-Claude Guillemin: Resources, Writing - review \& editing. Frank Lewen: Resources, Writing review \& editing. Stephan Schlemmer: Funding acquisition, Resources, Writing - review \& editing. 


\section{Declaration of competing interest}

The authors declare that they have no known competing financial interests or personal relationships that could have appeared to influence the work reported in this paper.

\section{Acknowledgments}

It is our great pleasure to dedicate this work to the memory of J.K.G. Watson for his many and invaluable contributions to the theory of high-resolution spectroscopy. We will also keep him in mind for his great sense of humor and for his Scottish accent.

We thank Arnaud Belloche for communicating results of his search for $c^{-13} \mathrm{CCH}_{4} \mathrm{O}$ in astronomical data. The work in Köln was supported by the Deutsche Forschungsgemeinschaft through the collaborative research center SFB 956 (project ID 184018867) project B3 and through the Gerätezentrum SCHL 341/15-1 ("Cologne Center for Terahertz Spectroscopy"). J.C. G. acknowledges support by the Centre National d'Etudes Spatiales (CNES; grant number 4500065585) and by the Programme National Physique et Chimie du Milieu Interstellaire (PCMI) of CNRS/INSU with INC/INP co-funded by CEA and CNES. Our research benefited from NASA's Astrophysics Data System (ADS).

\section{Appendix A. Supplementary Material}

Supplementary data associated with this article can be found, in the online version, at https://doi.org/101016/j.jms. ...

\section{References}

[1] J. E. Dickens, W. M. Irvine, M. Ohishi, M. Ikeda, S. Ishikawa, A. Nummelin, A. Hjalmarson, Detection of Interstellar Ethylene Oxide (c- $\left.\mathrm{C}_{2} \mathrm{H}_{4} \mathrm{O}\right)$, Astrophys. J. 489 (1997) 753-757. doi:10. 1086/304821

[2] A. Nummelin, J. E. Dickens, P. Bergman, A. Hjalmarson, W. M. Irvine, M. Ikeda, M. Ohishi, Abundances of ethylene oxide and acetaldehyde in hot molecular cloud cores, Astron. Astrophys. 337 (1998) 275-286.

[3] M. Ikeda, M. Ohishi, A. Nummelin, J. E. Dickens, P. Bergman, A. Hjalmarson, W. M. Irvine, Survey Observations of $\mathrm{c}-\mathrm{C}_{2} \mathrm{H}_{4} \mathrm{O}$ and $\mathrm{CH}_{3} \mathrm{CHO}$ toward Massive Star-forming Regions, Astrophys. J. 560 (2001) 792-805. doi:10.1086/322957

[4] M. A. Requena-Torres, J. Martín-Pintado, S. Martín, M. R. Morris, The Galactic Center: The Largest Oxygen-bearing Organic Molecule Repository, Astrophys. J. 672 (2008) 352-360. arXiv:0709.0542 doi:10.1086/523627
[5] J. M. Lykke, A. Coutens, J. K. Jørgensen, M. H. D. van der Wiel, R. T. Garrod, H. S. P. Müller, P. Bjerkeli, T. L. Bourke, H. Calcutt, M. N. Drozdovskaya, C. Favre, E. C. Fayolle, S. K. Jacobsen, K. I. Öberg, M. V. Persson, E. F. van Dishoeck, S. F. Wampfler, The ALMA-PILS survey: First detections of ethylene oxide, acetone and propanal toward the low-mass protostar IRAS 16293-2422, Astron. Astrophys. 597 (2017) A53. arXiv : 1611.07314 doi:10.1051/0004-6361/201629180

[6] A. Bacmann, A. Faure, J. Berteaud, Cold and Yet Complex: Detection of Ethylene Oxide in a Prestellar Core, ACS Earth Space Chem. 3 (6) (2019) 1000-1013. doi:10.1021/ acsearthspacechem.9b00072

[7] M. Berglund, M. E. Wieser, Isotopic compositions of the elements 2009 (IUPAC Technical Report), Pure Appl. Chem. 83 (2) (2011) 397-410. doi: $\{10.1351 /$ PAC-REP-10-06-02\}

[8] D. T. Halfen, N. J. Woolf, L. M. Ziurys, The ${ }^{12} \mathrm{C} /{ }^{13} \mathrm{C}$ Ratio in Sgr B2(N): Constraints for Galactic Chemical Evolution and Isotopic Chemistry, Astrophys. J. 845 (2) (2017) 158. doi:10.3847/1538-4357/aa816b

[9] H. S. P. Müller, A. Belloche, K. M. Menten, C. Comito, P. Schilke, Rotational spectroscopy of isotopic vinyl cyanide, $\mathrm{H}_{2} \mathrm{CCHCN}$, in the laboratory and in space, J. Mol. Spectrosc. $251(1-2)(2008)$ 319-325. arXiv:0806.2098 doi: $10.1016 / j \cdot j m s .2008 .03 .016$

[10] A. Belloche, H. S. P. Müller, R. T. Garrod, K. M. Menten, Exploring molecular complexity with ALMA (EMoCA): Deuterated complex organic molecules in Sagittarius B2(N2), Astron. Astrophys. 587 (2016) A91. arXiv:1511.05721 doi: 10.1051/0004-6361/201527268

[11] H. S. P. Müller, A. Belloche, L.-H. Xu, R. M. Lees, R. T. Garrod, A. Walters, J. van Wijngaarden, F. Lewen, S. Schlemmer, K. M. Menten, Exploring molecular complexity with ALMA (EMoCA): Alkanethiols and alkanols in Sagittarius B2(N2), Astron. Astrophys. 587 (2016) A92. arXiv:1512.05301 doi: 10.1051/0004-6361/201527470

[12] A. M. Jacob, K. M. Menten, H. Wiesemeyer, R. Güsten, F. Wyrowski, B. Klein, First detection of ${ }^{13} \mathrm{CH}$ in the interstellar medium, Astron. Astrophys. 640 (2020) A125. arXiv: 2007.01190 doi:10.1051/0004-6361/201937385

[13] T. L. Wilson, R. Rood, Abundances in the Interstellar Medium, Annu. Rev. Astron. Astrophys. 32 (1994) 191-226. doi:10. 1146/annurev.aa.32.090194.001203

[14] S. N. Milam, C. Savage, M. A. Brewster, L. M. Ziurys, S. Wyckoff, The ${ }^{12} \mathrm{C} /{ }^{13} \mathrm{C}$ Isotope Gradient Derived from Millimeter Transitions of $\mathrm{CN}$ : The Case for Galactic Chemical Evolution, Astrophys. J. 634 (2) (2005) 1126-1132. doi:10.1086/ 497123

[15] D. R. Schmidt, L. M. Ziurys, Exotic Carbon Chemistry in a Planetary Nebula: The Unusual Case of K4-47, Astrophys. J. Lett. 881 (2) (2019) L38. doi:10.3847/2041-8213/ab3663

[16] D. R. Schmidt, N. J. Woolf, T. J. Zega, L. M. Ziurys, Extreme ${ }^{13} \mathrm{C},{ }^{15} \mathrm{~N}$ and ${ }^{17} \mathrm{O}$ isotopic enrichment in the young planetary nebula K4-47, Nature 564 (7736) (2018) 378-381. doi: 10.1038/s41586-018-0763-1

[17] L. Margulès, A. Belloche, H. S. P. Müller, R. A. Motiyenko, J. C. Guillemin, R. T. Garrod, K. M. Menten, Spectroscopic study and astronomical detection of doubly ${ }^{13} \mathrm{C}$-substituted ethyl cyanide, Astron. Astrophys. 590 (2016) A93. arXiv: 1604.03256 doi:10.1051/0004-6361/201628309

[18] J. K. Jørgensen, H. S. P. Müller, H. Calcutt, A. Coutens, M. N. Drozdovskaya, K. I. Öberg, M. V. Persson, V. Taquet, E. F. van Dishoeck, S. F. Wampfler, The ALMA-PILS survey: isotopic composition of oxygen-containing complex organic molecules toward IRAS 16293-2422B, Astron. Astrophys. 620 (2018) A170. arXiv:1808.08753 doi:10.1051/ 
0004-6361/201731667

[19] J. Cernicharo, N. Marcelino, M. Agúndez, C. Bermúdez, C. Cabezas, B. Tercero, J. R. Pardo, Discovery of $\mathrm{HC}_{4} \mathrm{NC}$ in TMC-1: A study of the isomers of $\mathrm{HC}_{3} \mathrm{~N}, \mathrm{HC}_{5} \mathrm{~N}$, and $\mathrm{HC}_{7} \mathrm{~N}$, Astron. Astrophys. 642 (2020) L8. arXiv: 2009.07686 doi : 10.1051/0004-6361/202039274

[20] A. Bouchez, A. Walters, H. S. P. Müller, M. Ordu, F. Lewen, M. Koerber, S. Bottinelli, C. P. Endres, S. Schlemmer, Millimetre-wave spectrum of anti- ${ }^{13} \mathrm{C}_{1}$ and ${ }^{13} \mathrm{C}_{2}$ isotopologues of ethanol, J. Quant. Spectrosc. Radiat. Transfer 113 (11) (2012) 1148-1154. doi:10.1016/j.jgsrt.2012.02.025

[21] L. Margulès, R. A. Motiyenko, V. V. Ilyushin, J. C. Guillemin, Millimeter and submillimeter wave spectra of mono- ${ }^{13} \mathrm{C}$ acetaldehydes, Astron. Astrophys. 579 (2015) A46. doi:10. 1051/0004-6361/201425478

[22] R. A. Motiyenko, L. Margulès, V. V. Ilyushin, I. A. Smirnov, E. A. Alekseev, D. T. Halfen, L. M. Ziurys, Millimeter and submillimeter wave spectra of ${ }^{13} \mathrm{C}$ methylamine, Astron. Astrophys. 587 (2016) A152. doi:10.1051/0004-6361/ 201526924

[23] L. Kolesniková, Z. Kisiel, E. R. Alonso, J. C. Guillemin, J. L. Alonso, I. R. Medvedev, M. Winnewisser, A Comprehensive Spectral Rotational Analysis of the Interstellar Methyl Isocyanate $\mathrm{CH}_{3} \mathrm{NCO}$, Astrophys. J. Suppl. Ser. 245 (2) (2019) 31 doi:10.3847/1538-4365/ab570d

[24] V. V. Ilyushin, O. Zakharenko, F. Lewen, S. Schlemmer, E. A Alekseev, M. Pogrebnyak, R. M. Lees, L.-H. Xu, A. Belloche, K. M. Menten, R. T. Garrod, H. S. P. Müller, Rotational spectrum of isotopic methyl mercaptan, ${ }^{13} \mathrm{CH}_{3} \mathrm{SH}$, in the laboratory and towards Sagittarius B2(N2), Can. J. Phys. 98 (6) (2020) 530-537. arXiv:1910.02259 doi:10.1139/ cjp-2019-0421

[25] H. S. P. Müller, A. Brahmi M., J.-C. Guillemin, F. Lewen, S. Schlemmer, Rotational spectroscopy of isotopic cyclopropenone, $c-\mathrm{H}_{2} \mathrm{C}_{3} \mathrm{O}$, and determination of its equilibrium structure, Astron. Astrophys. 647 (2021) A179. arXiv:2102. 03570 doi: 10.1051/0004-6361/202040088

[26] R. Guesten, C. Henkel, W. Batrla, $\mathrm{H}_{2}{ }^{12} \mathrm{CO} / \mathrm{H}_{2}{ }^{13} \mathrm{CO}$ ratios from molecular clouds near the galactic center., Astron. Astrophys. 149 (1985) 195-198.

[27] F. F. Gardner, J. B. Whiteoak, J. Reynolds, W. L. Peters, T. B. H Kuiper, First detection of the ${ }^{18} \mathrm{O}$ isotope of methanol., Mon Not. R. Astron. Soc. 240 (1989) 35P-40. doi:10.1093/ mnras/240.1.35P

[28] B. Tercero, L. Margulès, M. Carvajal, R. A. Motiyenko, T. R Huet, E. A. Alekseev, I. Kleiner, J. C. Guillemin, H. Møllendal, J. Cernicharo, Microwave and submillimeter spectroscopy and first ISM detection of ${ }^{18} \mathrm{O}$-methyl formate, Astron. Astrophys. 538 (2012) A119. doi:10.1051/0004-6361/201117072

[29] A. Belloche, R. T. Garrod, H. S. P. Müller, K. M. Menten, I. Medvedev, J. Thomas, Z. Kisiel, Re-exploring Molecular Complexity with ALMA (ReMoCA): interstellar detection of urea, Astron. Astrophys. 628 (2019) A10. arXiv : 1906.04614 doi:10.1051/0004-6361/201935428

[30] G. L. Cunningham, A. W. Boyd, R. J. Myers, W. D. Gwinn, W. I. Le Van, The Microwave Spectra, Structure, and Dipole Moments of Ethylene Oxide and Ethylene Sulfide, J. Chem. Phys. 19 (6) (1951) 676-685. doi:10.1063/1.1748331

[31] H. A. Stuart, Über die Temperaturabhängigkeit der Dielektrizitätskonstanten von Gasen und Dämpfen. II, Z. Phys. 51 (7-8) (1928) 490-510. doi : 10.1007/BF01327841

[32] C. Hirose, Microwave spectra and $r_{0}, r_{s}$, and $r_{m}$ structures of ethylene oxide, Bull. Chem. Soc. Japan 47 (6) (1974) 13111318. doi:10.1246/bcs j.47.1311

[33] C. Hirose, Laboratory Microwave Spectrum of Ethylene Oxide,
Astrophys. J. Lett. 189 (1974) L145. doi : 10.1086/181487

[34] R. A. Creswell, R. H. Schwendeman, Centrifugal distortion and oxygen-17 quadrupole coupling in ethylene oxide, Chem. Phys. Lett. 27 (1974) 521-524. doi:10.1016/0009-2614(74) 80295-2

[35] J. Pan, S. Albert, K. V. L. N. Sastry, E. Herbst, F. C. De Lucia, The Millimeter- and Submillimeter-Wave Spectrum of Ethylene Oxide (c- $\left.\mathrm{C}_{2} \mathrm{H}_{4} \mathrm{O}\right)$, Astrophys. J. 499 (1998) 517-519. doi: 10. 1086/305638

[36] C. Medcraft, C. D. Thompson, E. G. Robertson, D. R. T. Appadoo, D. McNaughton, The Far-infrared Rotational Spectrum of Ethylene Oxide, Astrophys. J. 753 (2012) 18. doi: 10.1088/ 0004-637X/753/1/18

[37] S. Albert, Z. Chen, K. Keppler, P. Lerch, M. Quack, V. Schurig, O. Trapp, The Gigahertz and Terahertz spectrum of monodeutero-oxirane (c- $\left.\mathrm{C}_{2} \mathrm{H}_{3} \mathrm{DO}\right)$, Phys. Chem. Chem. Phys. 21 (2019) 3669-3675. doi:10.1039/C8CP05311A

[38] J. M. Flaud, W. J. Lafferty, F. Kwabia Tchana, A. Perrin, $\mathrm{X}$. Landsheere, First high-resolution analysis of the $v_{15}, v_{12}, v_{5}$, $v_{10}$ and $v_{2}$ bands of oxirane, J. Mol. Spectrosc. 271 (1) (2012) 38-43. doi:10.1016/j.jms.2011.11.005

[39] F. Kwabia Tchana, J. M. Flaud, W. J. Lafferty, M. Ngom, Absolute line intensities for oxirane in the $11.4 \mu \mathrm{m}$ spectral region, Mol. Phys. 112 (12) (2014) 1633-1638. doi:10.1080/ 00268976.2013 .851811

[40] M. A. Martin-Drumel, J. van Wijngaarden, O. Zingsheim, F. Lewen, M. E. Harding, S. Schlemmer, S. Thorwirth, Millimeter- and submillimeter-wave spectroscopy of disulfur dioxide, OSSO, J. Mol. Spectrosc. 307 (2015) 33-39. doi: $10.1016 / \mathrm{j} \cdot \mathrm{jms} .2014 .11 .007$

[41] H. S. P. Müller, O. Zingsheim, N. Wehres, J.-U. Grabow, F. Lewen, S. Schlemmer, Rotational Spectroscopy of the Lowest Energy Conformer of 2-Cyanobutane, J. Phys. Chem. A 121 (38) (2017) 7121-7129. doi:10.1021/acs.jpca. $7 \mathrm{~b} 06072$

[42] H. S. P. Müller, F. Lewen, Submillimeter spectroscopy of $\mathrm{H}_{2} \mathrm{C}^{17} \mathrm{O}$ and a revisit of the rotational spectra of $\mathrm{H}_{2} \mathrm{C}^{18} \mathrm{O}$ and $\mathrm{H}_{2} \mathrm{C}^{16} \mathrm{O}$, J. Mol. Spectrosc. 331 (2017) 28-33. arXiv: 1610 . 02174 doi:10.1016/j.jms.2016.10.004

[43] H. S. P. Müller, A. Maeda, S. Thorwirth, F. Lewen, S. Schlemmer, I. R. Medvedev, M. Winnewisser, F. C. De Lucia, E. Herbst, Laboratory spectroscopic study of isotopic thioformaldehyde, $\mathrm{H}_{2} \mathrm{CS}$, and determination of its equilibrium structure, Astron. Astrophys. 621 (2019) A143. arXiv:1812.01554 doi:10. 1051/0004-6361/201834517

[44] K. Tanaka, T. Tanaka, I. Suzuki, Dipole moment function of carbonyl sulfide from analysis of precise dipole moments and infrared intensities, J. Chem. Phys. 82 (7) (1985) 2835-2844. doi:10.1063/1.448285

[45] J. G. Lahaye, R. Vandenhaute, A. Fayt, $\mathrm{CO}_{2}$ laser saturation Stark spectra and global Stark analysis of carbonyl sulfide, J. Mol. Spectrosc. 119 (2) (1986) 267-279. doi:10.1016/ 0022-2852(86) 90023-8

[46] H. S. P. Müller, S. Thorwirth, D. A. Roth, G. Winnewisser, The Cologne Database for Molecular Spectroscopy, CDMS, Astron. Astrophys. 370 (2001) L49-L52. doi : 10.1051/0004-6361: 20010367

[47] H. S. P. Müller, F. Schlöder, J. Stutzki, G. Winnewisser, The Cologne Database for Molecular Spectroscopy, CDMS: a useful tool for astronomers and spectroscopists, J. Mol. Struct. 742 (13) (2005) 215-227. doi:10.1016/j.molstruc.2005.01. 027

[48] C. P. Endres, S. Schlemmer, P. Schilke, J. Stutzki, H. S. P. Müller, The Cologne Database for Molecular Spectroscopy, CDMS, in the Virtual Atomic and Molecular Data Centre, 
VAMDC, J. Mol. Spectrosc. 327 (2016) 95-104. arXiv: 1603. 03264 doi:10.1016/j.jms.2016.03.005

[49] H. M. Pickett, The fitting and prediction of vibration-rotation spectra with spin interactions, J. Mol. Spectrosc. 148 (2) (1991) 371-377. doi:10.1016/0022-2852(91) 90393-0

[50] K. M. T. Yamada, S. Klee, Pure Rotational Spectrum of $\mathrm{H}_{2} \mathrm{~S}$ in the Far-Infrared Region Measured by FTIR Spectroscopy, J. Mol. Spectrosc. 166 (2) (1994) 395-405. doi:10.1006/jmsp. 1994.1204

[51] L. Margulès, R. A. Motiyenko, J. Demaison, Millimeterwave and submillimeterwave spectra of sulfur dioxide ${ }^{32} \mathrm{~S}^{16} \mathrm{O}^{18} \mathrm{O}$ and ${ }^{32} \mathrm{~S}^{18} \mathrm{O}_{2}$, centrifugal distortion analysis and equilibrium structure, J. Quant. Spectrosc. Radiat. Transfer 253 (2020) 107153. doi: $10.1016 / j \cdot j q s r t .2020 .107153$

[52] J. K. G. Watson, Aspects of quartic and sextic centrifugal effects on rotational energy levels, in: J. R. Durig (Ed.), Vibrational spectra and structure, 6, Elsevier; Amsterdam, 1977, pp. 1-89.

[53] L. Margulès, R. A. Motiyenko, E. A. Alekseev, J. Demaison, Choice of the reduction and of the representation in centrifugal distortion analysis: A case study of dimethylsulfoxide, J. Mol. Spectrosc. 260 (1) (2010) 23-29. doi :10.1016/j.jms. 2009. 12.009 